(G) INTERPLANETARY MISSIONS 


\title{
FUTURE SOLAR SYSTEM MISSIONS
}

\author{
GEOFFREY A. BRIGGS \\ Solar System Exploration Division, Office of Space Science and Applications NASA \\ Headquarters, Washington, D.C.
}

After a decade long hiatus in launches beyond Earth orbit, NASA's planetary exploration program is again moving forward, beginning with the Magellan launch to Venus in May 1989 and the Galileo launch to Jupiter in October 1989. These spacecraft will reach their targets in August 1990 and December 1995, respectively. Both are missions of longstanding priority, Magellan to provide the first global high resolution mapping of the cloud-shrouded Venus surface, Galileo to make comprehensive measurements of the Jovian system in follow-up to the 1979/1980 Voyager flybys.

Beyond these two missions there are other already approved missions: the Mars Observer for launch in 1992, the Comet Rendezvous and Asteroid Flyby CRAF mission (Fig. 1) for launch in 1995, and the Cassini mission (Figs. 2 and 3) to Saturn and its moon Titan for launch in 1996. The very diversity of these five missions and their targets (Venus, the Jovian system, Mars, comet Kopff, asteroids Gaspra and Ida by Galileo, Hamburga by $C R A F$, and Maja by Cassini, and the Saturnian system, is indicative of the strategy being pursued in the program: one of deliberate breadth that seeks to explore all three main classes of solar system bodies (the terrestrial planets, the outer giants and their moons, and the primitive small bodies).

This broadly-based strategy, as opposed to a focus on, say, Mars and Venus only, was developed in response to recommendations by the National Academy of Sciences and is serving the nation well in that it provides to the science community a comprehensive perspective from which to interpret new results and fit them into our growing body of knowledge: also, the continuing stream of images of the new worlds of the solar system evokes a worldwide public interest, as evidenced by the tremendous impact of the Voyager encounters of the last decade.

The future direction of the program beyond those missions mentioned above, which are already approved, is beginning to take shape as a result of a continuing strategic planning exercise begun a year ago (Fig. 4). This exercise directly involves the science community and the several NASA Centers with an interest in the program; the planning is conducted using as a guide the general principles embodied in the NASA Office of Space Science and Applications Strategic Plan, and it necessarily calls for iteration as the overall NASA program continues to take shape. Specifically, since 20 July 1989, when President Bush announced his commitment to the human exploration of the Moon and Mars, planning for the future robotic exploration of those bodies has taken on a new significance - that of providing appropriate precursor knowledge, both scientific and technological, to lay the ground work for the humans who will follow.

Unlike the Moon, where the Apollo program has clearly established the feasi-

Y. Kondo (ed.), Observatories in Earth Orbit and Beyond, 297-306.

(C)1990 Kluwer Academic Publishers. Printed in The Netherlands. 
bility of human exploration and where there is only one required precursor mission (a polar orbiter to provide a global data base of topographic and geochemistry information; see Figs. 5-7), in the case of Mars much more needs to be done to establish the groundwork - certain essential technologies need proving and a good deal of basic information about Mars (Fig. 8) must be gathered, specifically: surface and atmospheric hazards; the availability of water; knowledge relevant to the issues of planetary protection; and knowledge of the most interesting sites for scientific exploration.

Already a sequence of robotic Mars missions (Fig. 9) has been identified (not much different from the series of science missions long since recommended by the NAS-Space Science Board for the in-depth exploration of Mars) and is being refined in consultation with the Space Exploration Initiative planning team at the Johnson Space Center. These missions include a surface network of small, rugged rough landers to observe the character of a large number of surface sites for future landings (Fig. 10) and to measure the seismic charter of Mars and monitor the atmospheric dynamics. Then surface sample return missions, field geology rovers and high resolution imaging orbiters are required along with a communication satellite infrastructure to support this extended effort. The pace and overall scope of this effort will be defined over the next several years.

As noted earlier, an essential characteristic of the solar system exploration program to date has been breadth; how does one retain that breadth while focussing on the Moon and Mars as national space priorities? First, already-approved missions will continue through about 2005, returning data from the depths of the solar system. Second, other opportunities present themselves: to use ground based and Earth orbital telescopes to search for and characterize planetary systems associated with other stars, and to use newly emerging "lightsat" and microsatellite technologies to inexpensively complete the reconnaissance of our own Solar System (even after Voyager we still have not visited Pluto/Charon or Chiron or the mainbelt and Earth-approaching asteroids).

Planning to begin a serious, deep search for other planetary systems is presently focussed on the application of various approaches to ultra-high precision astrometry (to look for the reflex motion of stars resulting from the orbital motion of associated planets) and the possible use of space-based coronagraphic telescopes (similar in principle to the one used at Cerro Tolollo to discover the dust disk about the star Beta Pictoris). To be effective in observing individual planets near a star of enormously greater brightness, such coronagraphic telescopes would need to have ultra-low light scattering mirrors (super smooth mirror technology is advancing apace because of the needs of the photo-lithography business). In both cases a good deal of technology development lies ahead but one may confidently expect such observatories to be available around the turn of the century; the results from such observing programs may well revolutionize our thinking about our own Solar System and will, assuredly, give us much greater confidence in those theories of planetary origin and evolution that stand the test of being applied to other solar systems.

The prospects of an inexpensive "Explorer-like" program of solar system reconnaissance missions are being assessed. A key ground rule is that launch vehicles 
must be small - not larger than a Delta - since there is a clear association of spacecraft mass (and consequent complexity) and mission cost. Already the feasibility of launching a $300 \mathrm{~kg}$ (dry) spacecraft on a delta-VEGA/Jupiter swingby trajectory to Pluto using a Delta launch vehicle has been established; such a spacecraft with a moderately comprehensive science payload of remote sensing and particle and fields instrumentation could reach Pluto within 15 years of a 1998 launch; such a flyby would allow observations of the transient Pluto atmosphere - which has apparently caused Pluto to brighten dramatically over the last twenty years as it passes through the perihelion part of its orbit - before the atmosphere freezes out again around the year 2020. For missions to the Earth-approaching asteroids even smaller spacecraft are contemplated - taking advantage of microspacecraft technology developments that have been made over the last several years. Here launchers like the DARPA's planned Taurus vehicle or equivalent are contemplated, a vehicle significantly smaller than the Delta but larger than the existing Scout and Pegasus vehicles. Mission goals would inevitably have to be quite focused but the range of critical characteristics that can be measured with only a few key instruments is impressive and sensor technologies are creating highly sensitive solid state devices that do not require large telescopic optics as in the past.

Thus there is optimism that an innovative, low cost planetary mission capability - the "Discovery Program" - will emerge over the next few years to maintain scientific balance in the solar system exploration program even as the program focuses on satisfying the requirements of the Space Exploration Initiative. Such a program might well also assume the competitive character of the Explorers where a Principal Investigator proposes the mission and, upon selection, is given the authority and responsibility to carry out the mission in association with an appropriate NASA Center. 


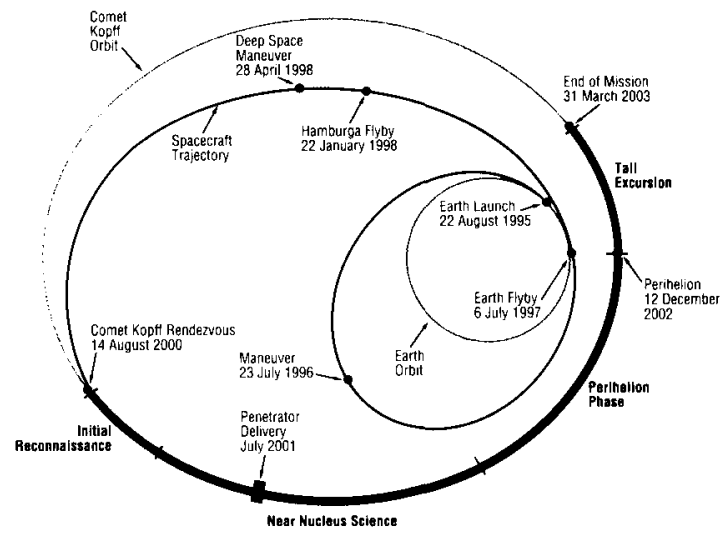

Fig. 1. Trajectory and mission phases of CRAF.

2u1! jo \%001 a:

s7sop

PFI:A :

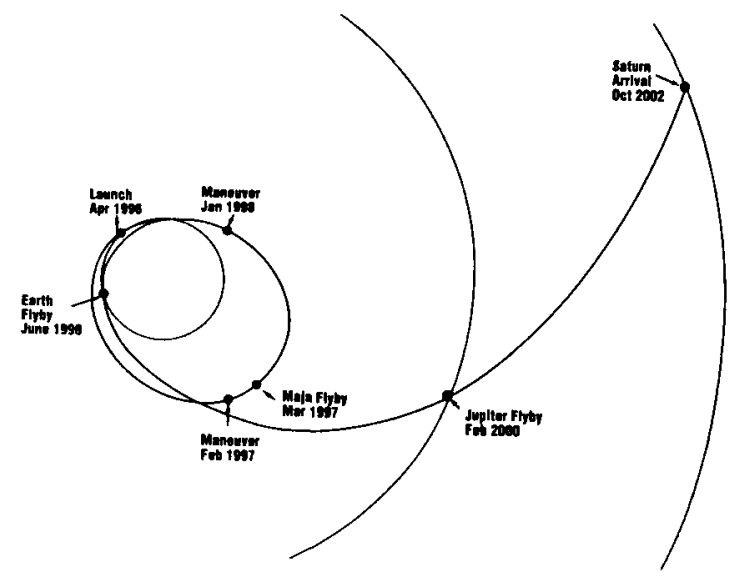

Fig. 2. The interplanetary trajectory of the Cassini spacecraft.

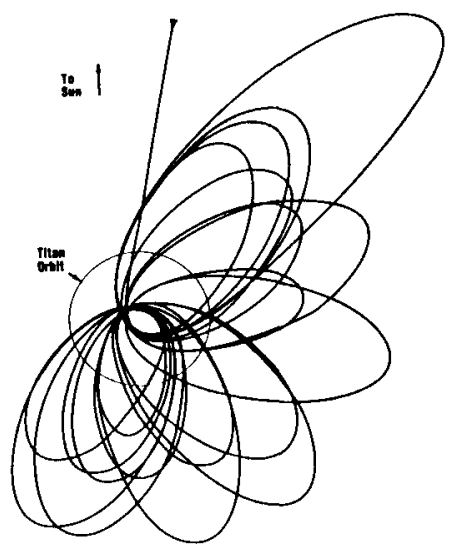

Fig. 3. The Cassini trajectory after arrival at Saturn, passing Titan on each orbit. 


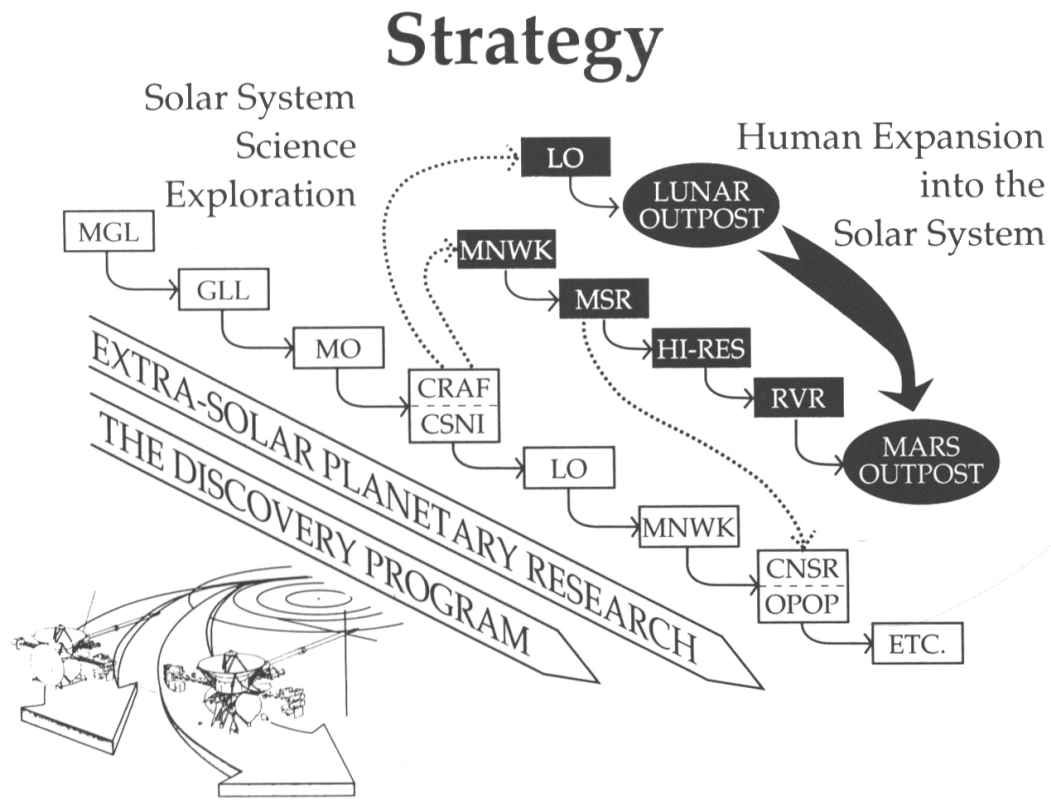

Fig. 4. Strategic Plan for the Exploration of the Solar System.

\section{Lunar Outpost Precursor Missions}

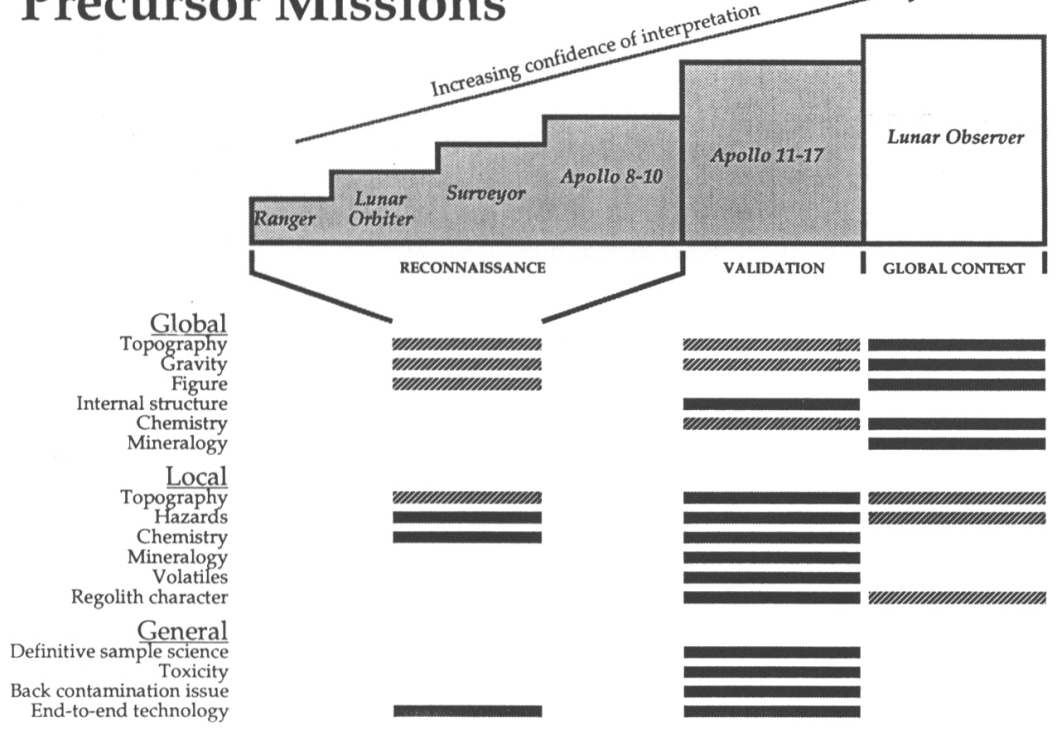

Fig. 5. Lunar Outpost Precursor Missions. 


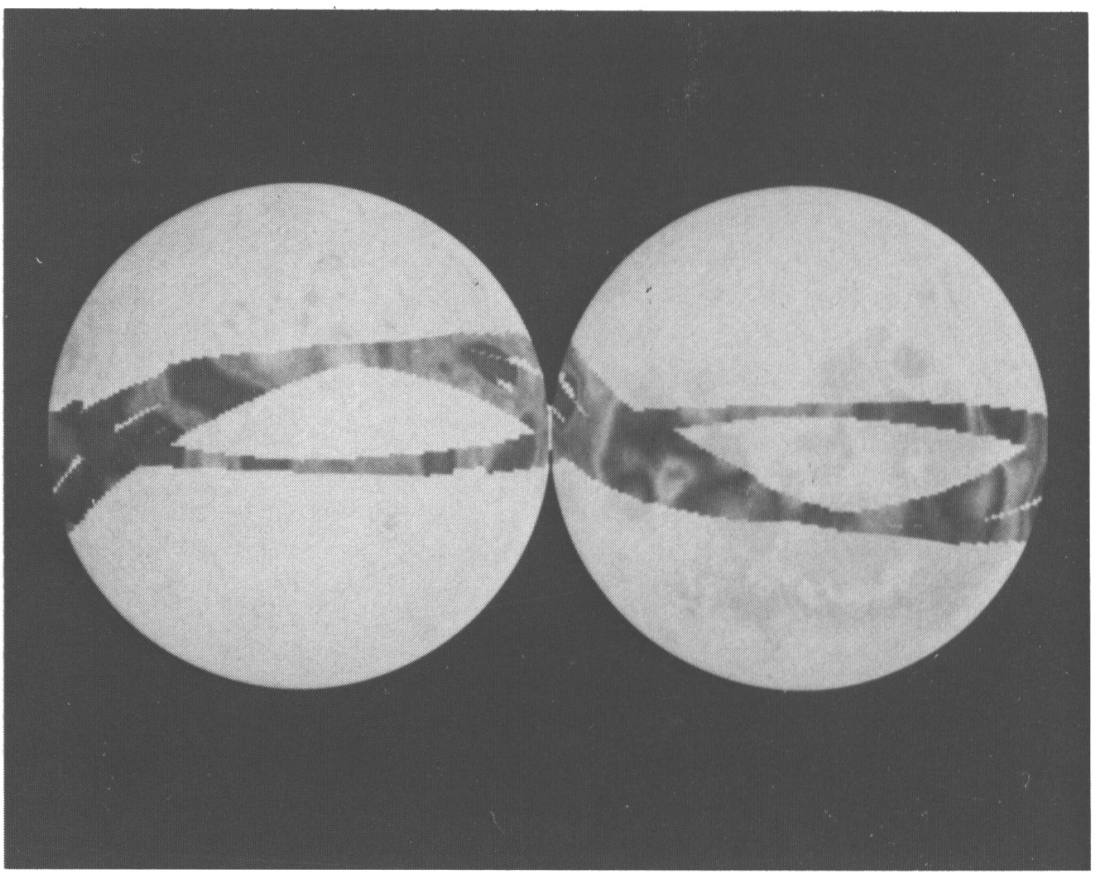

Fig. 6. The bands superimposed on this photograph of the front and back of the Moon represent the only areas of the lunar surface that have been chemically analyzed. The remaining 80 percent is still almost completely unknown. 


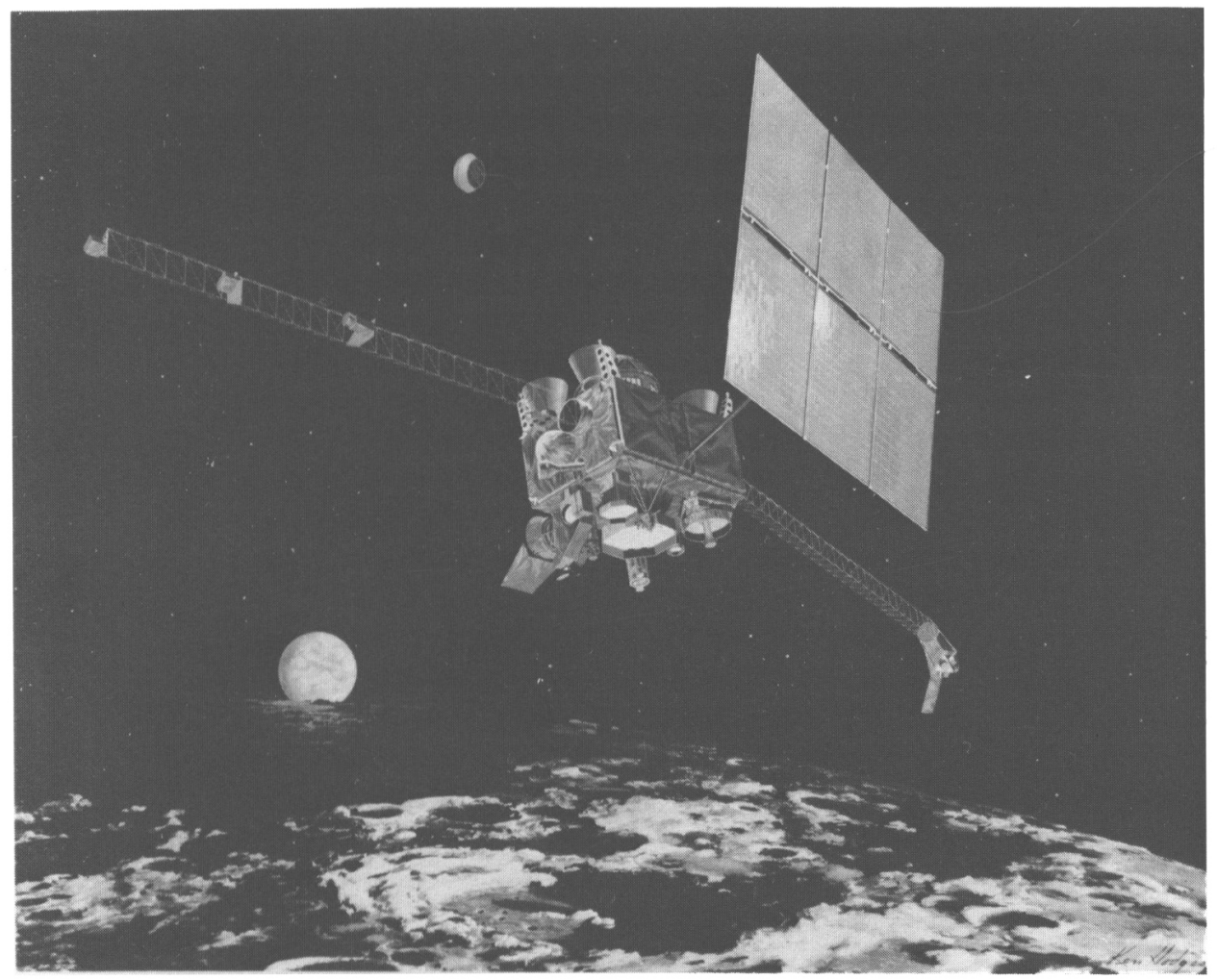

Fig. 7. An artist's concept of the Lunar Observer mapping the lunar surface. The data the Observer collects will help planners select a site for a manned lunar base and determine what human activities can be accomplished, as well as advancing scientific knowledge of our closest neighbor. 


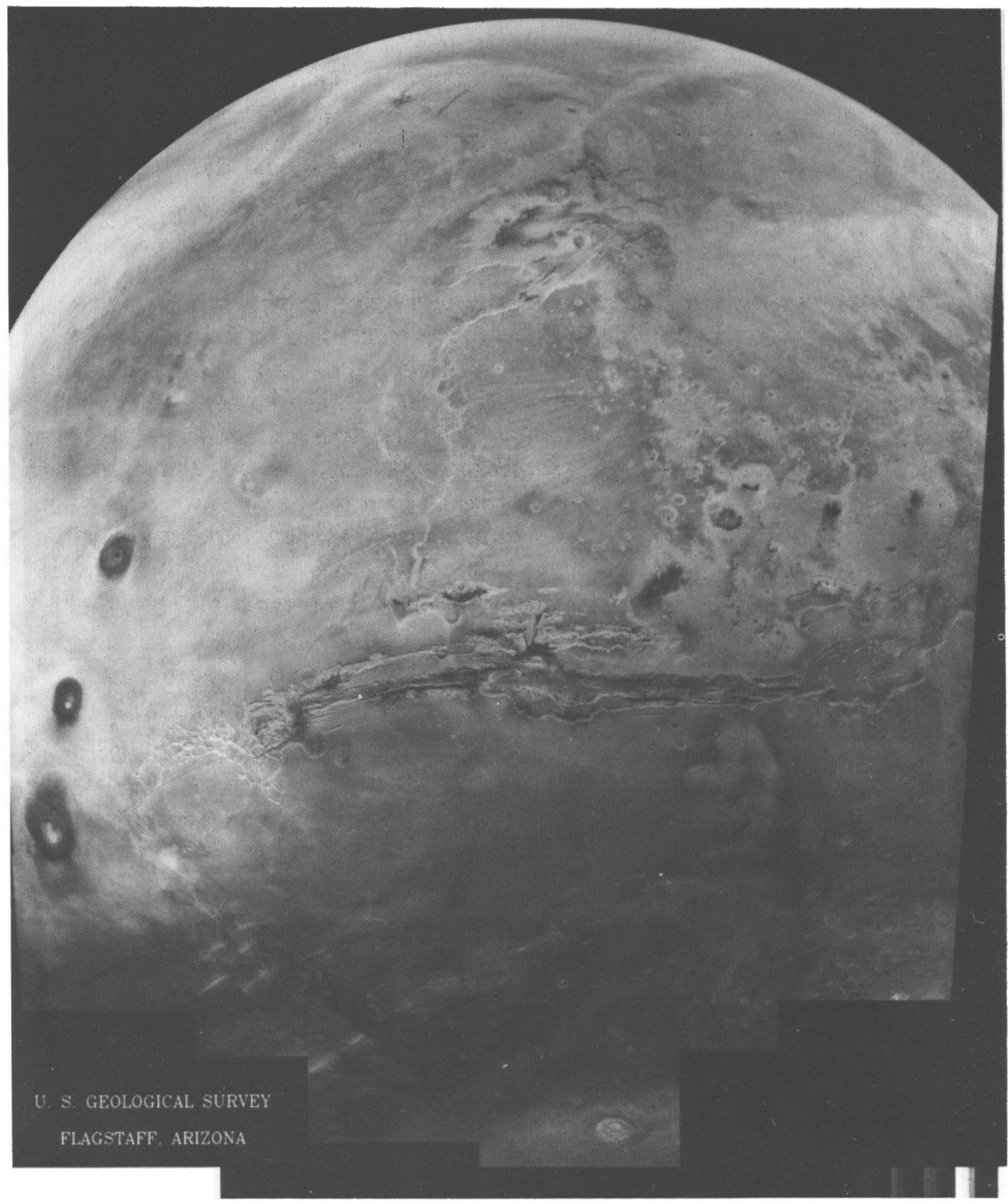

Fig. 8. Mosaic of nearly an entire hemisphere of Mars. 


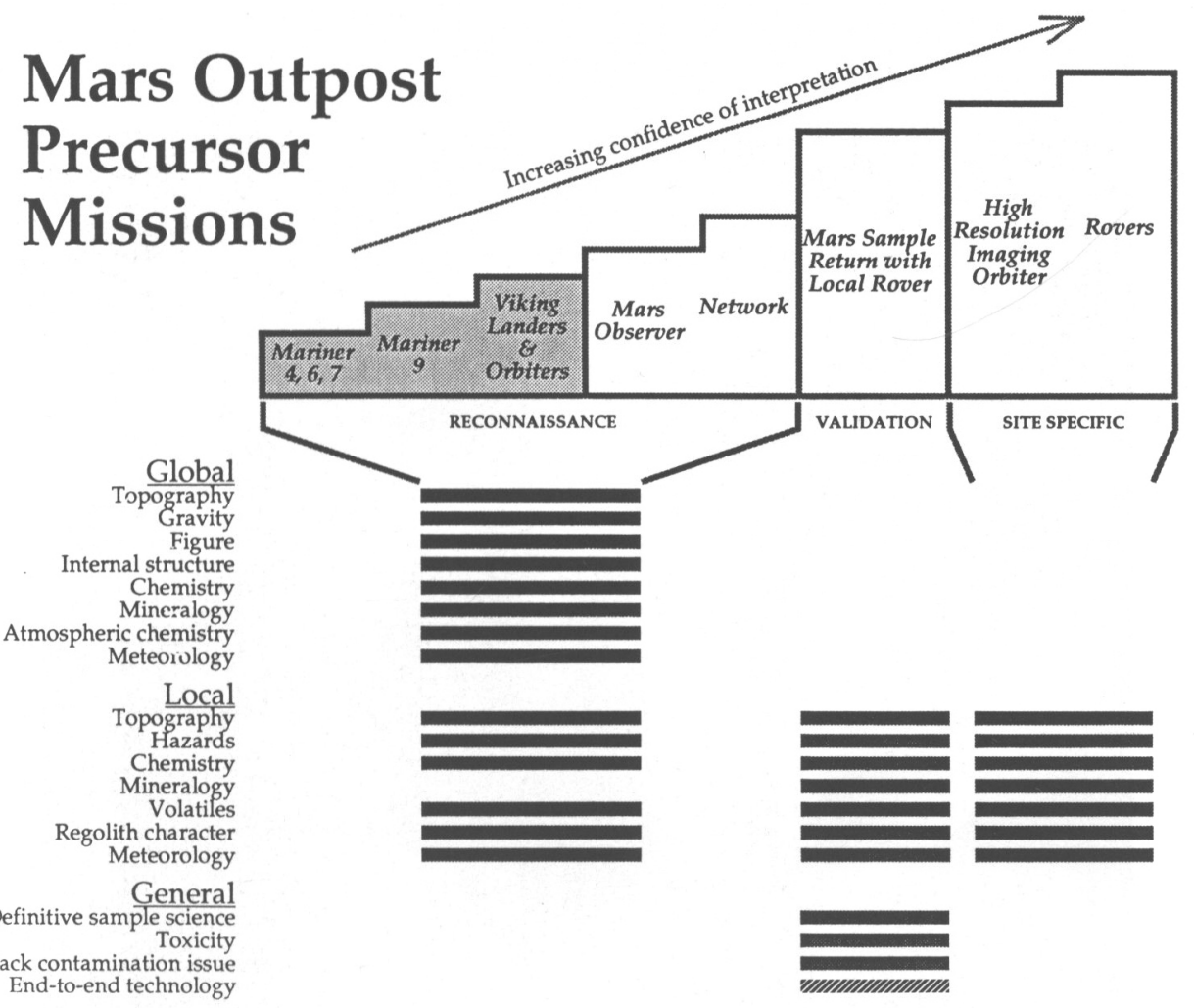

Fig. 9. Mars Outpost Precursor Missions. 


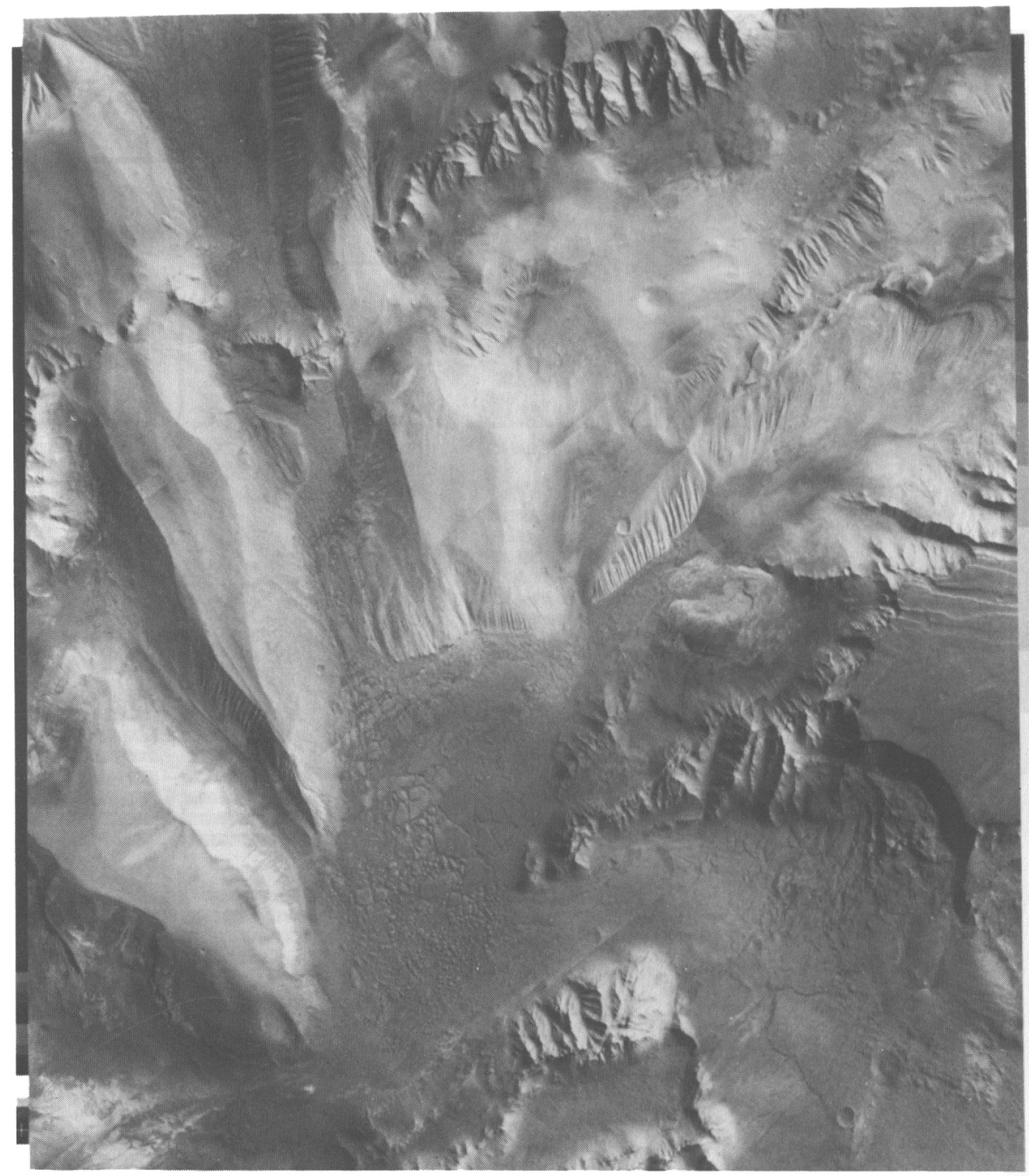

Fig. 10. Detail of Mars' Valles Marineris equatorial rift system, a possible future sample return and landing site. 